\title{
QoS Adaptation in Multimedia Multicast Conference Applications for E-Learning Services
}

\author{
Sérgio Deusdado, Instituto Politécnico de Bragança, Portugal \\ Paulo Carvalho, Universidade do Minho Braga, Portugal
}

\begin{abstract}
The evolution of the World Wide Web service has incorporated new distributed multimedia conference applications, powering a new generation of e-learning development and allowing improved interactivity and prohuman relations. Groupware applications are increasingly representative in the Internet home applications market, however, the Quality of Service (QoS) provided by the network is still a limitation impairing their performance. Such applications have found, in multicast technology, an ally contributing for their efficient implementation and scalability. Additionally, considering $Q o S$ as a design goal at the application level becomes crucial for groupware development, enabling QoS productivity to applications. The applications' ability to adapt themselves dynamically according to the resources availability can be considered a quality factor.Tolerant real-time applications, such as video conferences, are in the frontline to benefit from QoS adaptation. However, not all include adaptive technology able to provide both end-system and network quality awareness. Adaptation, in these cases, can be achieved by introducing a multiplatform middleware layer responsible for tutoring the applications' resources (enabling adjudication or limitation) based on the available processing and networking capabilities. Congregating these technological contributions, an adaptive platform has been developed integrating public domain multicast tools, applied to a Web-based distance learning system. The system is user-centered (e-student), aiming at good pedagogical practices and proactive usability for multimedia and network resources. The services provided, including QoS adapted interactive multimedia multicast conferences (MMC), are fully integrated and transparent to end-users. QoS adaptation, when treated systematically in tolerant real-time applications, denotes advantages in group scalability and QoS sustainability in heterogeneous and unpredictable environments such as the Internet.
\end{abstract}

Keywords: e-learning; multicast; groupware; QoS adaptation

\section{INTRODUCTION}

Technology has been a strong catalyst for educational innovation and improvement, espe- cially when the World Wide Web is involved. The next generation Internet needs technological support to accommodate promising new applications, such as interactive real-time

Copyright (C) 2006, Idea Group Inc. Copying or distributing in print or electronic forms without written permission of Idea Group Inc. is prohibited. 
multimedia distribution. Predictable bandwidth availability and capacity solvency imply QoS management to regulate resources in heterogeneous environments. Actually, increasing the network capacity through advanced network and media technology is not per se a ubiquitous and definitive solution to overcome the network capacity problem. Historically, the users have always managed to consume the entire system capacity soon after it was enlarged (Ferguson \& Huston, 1998). IP Multicasting techniques (Deering, 1998; Kosiur, 1998; Moshin, Wong, \& Bhutt, 2001; Thaler \& Handley, 2000) are attractive solutions for this capacity shortage problem as bandwidth consumption is reduced when network resources are shared. On the other hand, the QoS support (Moshin, Wong, \& Bhutt, 2001) should be, in a first instance, inherent to applications in order to integrate conveniently enhanced real-time multimedia applications in the present Internet, barely QoS aware and increasingly heterogeneous.

With the advent of wireless and mobile networks, heterogeneity is likely to subsist; envisioned applications should merge QoS adaptation and multicast in a proactive utilization of resources. Applications should be designed with adaptation in mind; they need to employ built-in mechanisms that allow them to probe the conditions of the network environment and alter their transmission characteristics accordingly (Miras, 2002). Self-adaptive applications, in the sense of proactive behavior for transmission of continuous media in multiparty applications, are a well-accepted solution due to the correct integration of new services in today's Internet (Deusdado, 2002; Li, Xu, Naharstedt, \& Liu, 1998).

E-learning, as a component of flexible learning, encompasses a wide set of applications and processes which use available electronic media to deliver vocational education and training. It includes computer-based learning, Web-based learning, virtual classrooms and digital collaboration (Eklund, Kay, \& Lunch, 2003). Our work aims to integrate interactive multimedia e-learning applications in a proactive fashion taking into account the available network resources and QoS sustainability. In this way, our motivation is to offer improved learning experience based on ultimate technology with QoS warranties.

The system architecture proposed in this article includes an adaptive module based on Java applets and embedded Javascript, responsible for assessing the existing operating conditions, by collecting metrics reflecting the client's end-system performance (e-student's host), the current network conditions and relevant multicast group characteristics. The collected data are subsequently computed weighting parameters such as the available bandwidth at the client side, the round-trip time between the client and the e-learning server, the client's current CPU load and free memory. The obtained results are used for proper multicast applications scheduling and parameterization in a transparent way.

\section{MOTIVATIONS}

Basically, e-learning services are used to promote connections between people (e-students) and training resources (Steeples \& Jones, 2002). E-learning research is wide and growing in importance, especially in higher education. Several institutions are developing interactive Web-based learning systems, integrating rich media streaming which may compromise network performance. The design of e-learning systems should consider QoS as mandatory for successful learning experiences, selecting the appropriate technologies and applications, and regulating proactively the information and communications technology (ICT) resources utilization (Allison, Ruddle, McKechan \& Michaelson, 2001).

The Multicast Backbone (MBone) is a network overlaying the global Internet designed to support multipoint applications. MBone tools comprise a collection of audio, video and whiteboard applications that use Internet multicast protocols to enable multiway communications (point-to-multipoint and multipoint-to-multipoint), satisfying most of the needs of group communication, such as e-learning services. Using these applications by common e-students drives recurrently to poor QoS satisfaction due 
Figure 1. QoS tolerance for generic audio and video applications (Miras, 2002)

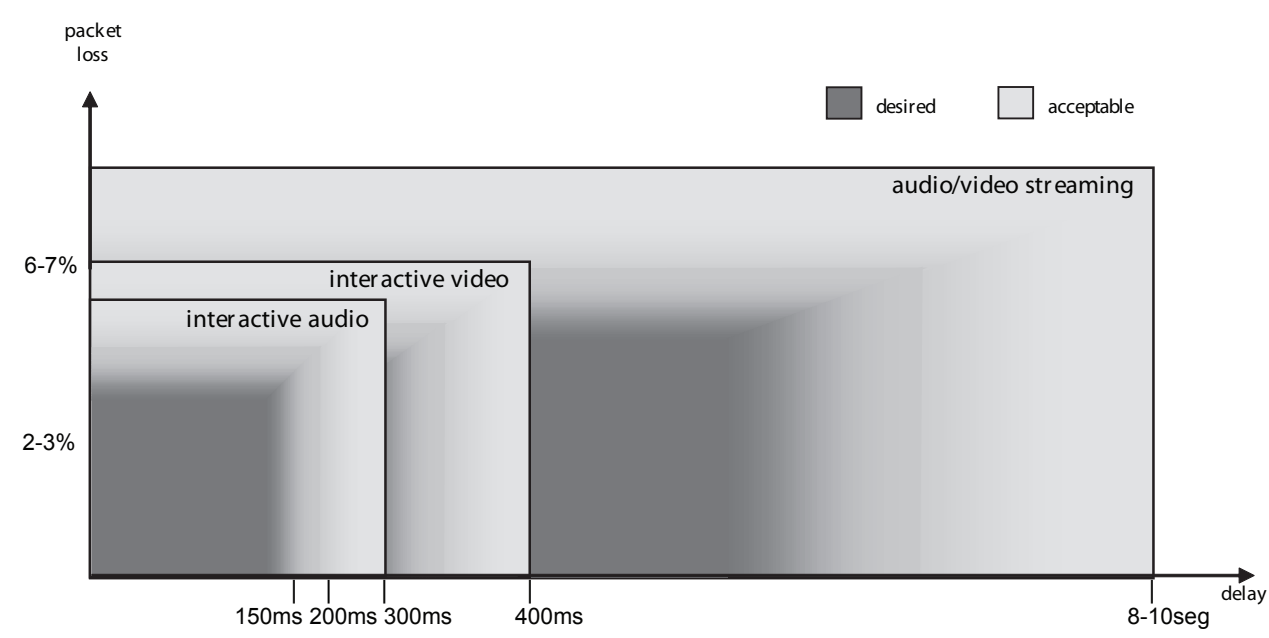

to the heterogeneity of resource conditions and the applications' inability to assess available conditions and adjust internal parameters before conference initiation. Without regulation, Realtime Transport Protocol (RTP) traffic floods the network capacity insensitively, forcing network congestion in certain cases or inhibiting better performance. A coherent behavior of an application without adaptation is difficult in today's Internet.

Public domain multicast applications used in this article, vic (McCanne \& Jacobson, 1995), rat (Hardman, Kirstein, Sasse, Handley \& Watson, 1995) and Java Media Framework (JMF) (JMF 2.0, 1999) were designed with no QoS “sensors," so the communication dynamics is not automatically interdependent of end-systems or network conditions. Effectively, such applications allow preparameterization to adjust critical parameters such as throughput, number of frames per second, video and audio encoding formats and so forth. Adaptation, in these cases, can be obtained by introducing a multiplatform middleware layer responsible for tutoring the applications' resources (adjudication or limitation) based on the available processing and networking capabilities (Miras, 2002).

Common interactive real-time applications are fault-tolerant but suffer from QoS constraints; low-latency requirements and reliability are cumulative to achieve conference success. The diagram in Figure 1 attempts to illustrate the QoS tolerance, in terms of delay and packet loss, for generic interactive audio and video applications.

The main motivation of this article is to provide adaptive behavior to applications used on both sides of multimedia conferencing, focusing essentially on multicast members that initiate audio and/or video transmission. The underlying idea is to launch automatically MMC applications with proper audio and video codecs, bandwidth allocation inference and other parameters that affect sustainability and scalability during an e-learning session. Our emphasis is on the concept of "interactive 
e-learning services," relegating the concept of "e-learning course" to a secondary goal, which will be considered in future work.

Most prominent related work on friendly multimedia transmission over the Internet, based on a combination of system and network QoS feedback implementing equation-based adaptation is summarized in Bouras and Gkamas (2003) and Vandalore, Feng Jain, and Fahmy (2001).

\section{SYSTEM'S ARCHITECTURE}

For multicast video distribution to heterogeneous users in an e-learning session, we assume that a class server (e-tutor's system) should be distributed and platform independent, considering inclusively multitutoring. Thus, a class server should connect to an e-learning server (Web server) and be submitted to adaptation as a regular new sender. The QoS requirements for the class server, operating in a centralized fashion, may justify the need of layered multicast (Johanson \& Lie, 2002; Liu, Li \& Zhang, 2004), enhancing the service's adaptation. However, this work aims at integrating e-students with heterogeneous equipment when they transmit audio and video to the group, as it happens in a conventional classroom. If a client (e-student) wants to interact and multicast video then the system's architecture will be integrated with fair adjustments attending both to the connection to the server (e-tutor) network and to the hardware processing capabilities. Client's adaptation should not depend on the other group members because they are transient, and consequently stability of transmissions could be very poor.

As the involved applications are characterized by an intensive use of host and network resources, the purpose of the middleware platform is to achieve by computation, on a scale of five differentiated modes, the proper integration of new multicasting members. Within this thematic, it means implementing an adaptive learners' participation in e-learning sessions by starting MMC applications transparently, with their functionality optimized for the current operating conditions.
To clarify these aspects Figure 2 illustrates the system architecture. As shown, three applets, operating sequentially and interdependently, are responsible for monitoring and assessing QoS conditions, inferring, announcing and/or editing computed adaptation parameters. The process culminates with the initiation of MMC applications, depending on the host and network profiles and covering eventual end-user explicit requirements.

Audio and video encoding formats, frame rate and other quality metrics may be chosen according to the resources' availability, providing coherent, friendly and fair participation in the network load balance. After monitoring sustainable network QoS with repeated measurements during approximately 15 seconds before media transmission, the Round-Trip Time (RTT) and bandwidth are calculated using a moving average. In addition, system's status variables, such as processor load, free memory, processor performance and so forth, are acquired, taking advantage of operating system facilities.

The system is multiplatform as the included applets differentiate the most popular operative systems (Windows and Unix), invoking appropriate inner services to obtain instant measures for the processor's load and free memory. The collected data constitutes another input to compute an adaptation index. Different compilations were produced for common browsers.

All of the adaptation process is transparent, however, regarding the experimental nature of this work, each phase allows interaction with the user, providing technical information or even accepting user preferences. To achieve this goal, applets and HTML forms interchange data using Sun's Liveconnect technology.

\section{ADAPTIVE QOS FRAMEWORK}

In the proposed framework, QoS management is performed individually for each new conference member and occurs before the transmission's start, such that MMC applications are launched adaptively facing the previous QoS sensing period conditions. QoS variability during the conference is not used to dynami- 
Figure 2. System's architecture

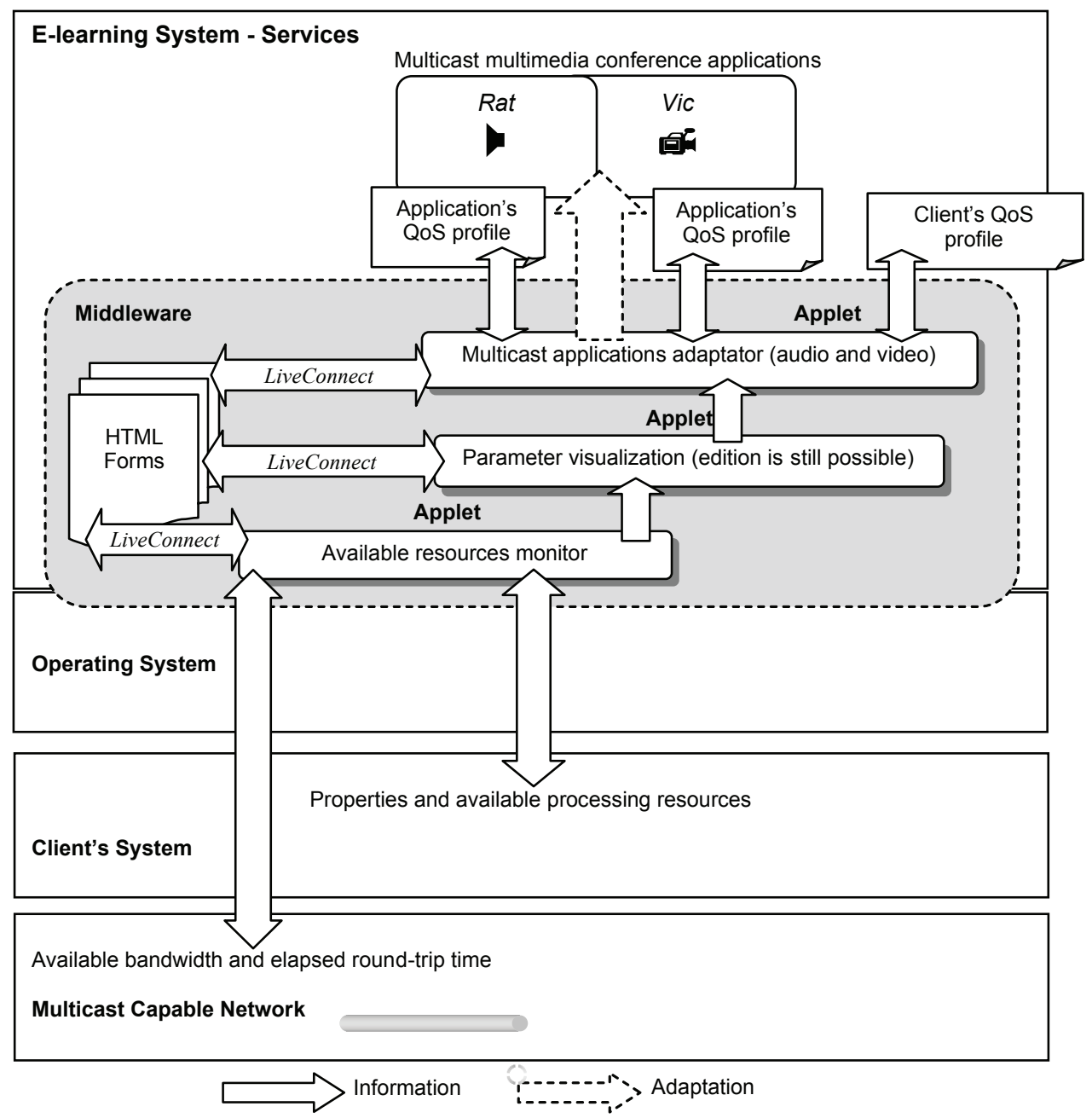

cally readapt the applications. If an e-student experiences lack of QoS while conferencing, the membership process should be restarted. Corroborating this practice, MMC applications, especially vic, are not stable enough. In fact, if some critical adjustments are made on-the-fly, the result is often the collapse of the application. Nevertheless, dynamic adaptation is currently a subject of study within group communication applications (Layaida \& Hagimonte, 2002;
Tusch, Böszörményi, Goldschmidt, Hellwagner \& Schojer, 2004).

Considering the applications' specificity and type of traffic generated, adaptability only includes interactive audio (rat) and video (vic) applications and services. The heuristics regarding the choice of applications' QoS parameters emerged from experimental results and scientific references in this matter (Wu, Hou, Zhu, Zhang, \& Peha, 2001). For instance, video 
Table 1. vic and rat QoS parameters used to adjust applications' profile.

\begin{tabular}{|l|l|l|}
\hline$R a t$ & $\mathbf{- f}$ format & Indicates audio encoding format: $l 16, p c m, d v i, g s m$ and $l p c$ \\
\hline Vic & $\mathbf{- B} k b p s$ & Sets the maximum bandwidth slider (kbps) \\
\hline Vic & $\mathbf{- c}$ dither & $\begin{array}{l}\text { On a color-mapped display, uses the algorithm indicated by dither (e.g., } e d, \\
\text { gray, } o d, \text { quantize }) \text { to convert to the available color palette }\end{array}$ \\
\hline Vic & $\mathbf{- f}$ format & Indicates the video encoding format: $h 261, h 263, j p e g, n v, \ldots$ \\
\hline Vic & $\mathbf{- F} f p s$ & Sets the maximum frame rate (fps) \\
\hline
\end{tabular}

Table 2. Set of parameters for different QoS adaptation modes

\begin{tabular}{|c|c|c|c|c|c|}
\hline $\begin{array}{c}\text { ADAPTATION } \\
\text { MODE }\end{array}$ & $\begin{array}{c}\text { MAXIMUM } \\
\text { BANDWIDTH }\end{array}$ & $\begin{array}{c}\text { FRAME } \\
\text { RATE }\end{array}$ & $\begin{array}{c}\text { VIDEO } \\
\text { CODEC }\end{array}$ & COLOR & $\begin{array}{c}\text { AUDIO } \\
\text { CODEC }\end{array}$ \\
\hline 5 & $1 \mathrm{Mbps}$ & $30 \mathrm{fps}$ & H.261 & Yes & L16 \\
\hline 4 & $512 \mathrm{kbps}$ & $25 \mathrm{fps}$ & H.261 & Yes & PCM \\
\hline 3 & $256 \mathrm{kbps}$ & $20 \mathrm{fps}$ & H.261 & Yes & DVI \\
\hline 2 & $128 \mathrm{kbps}$ & $15 \mathrm{fps}$ & H.263 & Yes & GSM \\
\hline 1 & $64 \mathrm{kbps}$ & $10 \mathrm{fps}$ & H.263 & No & LPC \\
\hline
\end{tabular}

conference users typically require better audio quality than video quality (Bolot, Crépin, \& Garcia 1995). The success of video conferencing communication also depends on factors such as received frames per second, image quality, resolution, size and illumination.

For this work, the representative parameters of vic and rat used to modulate QoS are presented in Table 1.

The values for these parameters, deriving from a mathematical expression that generates an adaptation mode based on the sustainable QoS level, compose a set of adjusting directives determining the applications' behavior. Each adaptation mode indexes the respective set of adjustments, which will then be passed to the application. Since QoS scale varies from mode 1 to 5 , when the obtained result is under or over this range it will be assigned to the nearest limit. Equation (1) determines the adaptation mode to be applied:

$$
\mathrm{M}=(\text { int })(\mathrm{B} /(\mathbf{R T T} / 2)+\mathrm{FM} / \mathrm{P}) * \mathrm{~K}
$$

where ${ }^{1}$,

$\mathbf{M}=$ QoS adaptation Mode (Table 2);

$\mathbf{B}=$ Bandwidth (kbps); RTT = Round-Trip Time (ms);

$\mathbf{F M}=$ Free Memory (MB); $\mathbf{P}=$ Processor load (\%);

$\mathbf{K}=1 / 50$ - constant to scale the result ( 1 to 5).

For vic (version 2.8), the video encoding formats H.261 (ITU-T H.261, 1993) and H.263 (ITU-T H.263, 1998) were those who revealed best performance for e-learning purposes, lead- 
Figure 3. E-learning system screenshots and MMC's adaptation HTML forms based on available $Q o S$

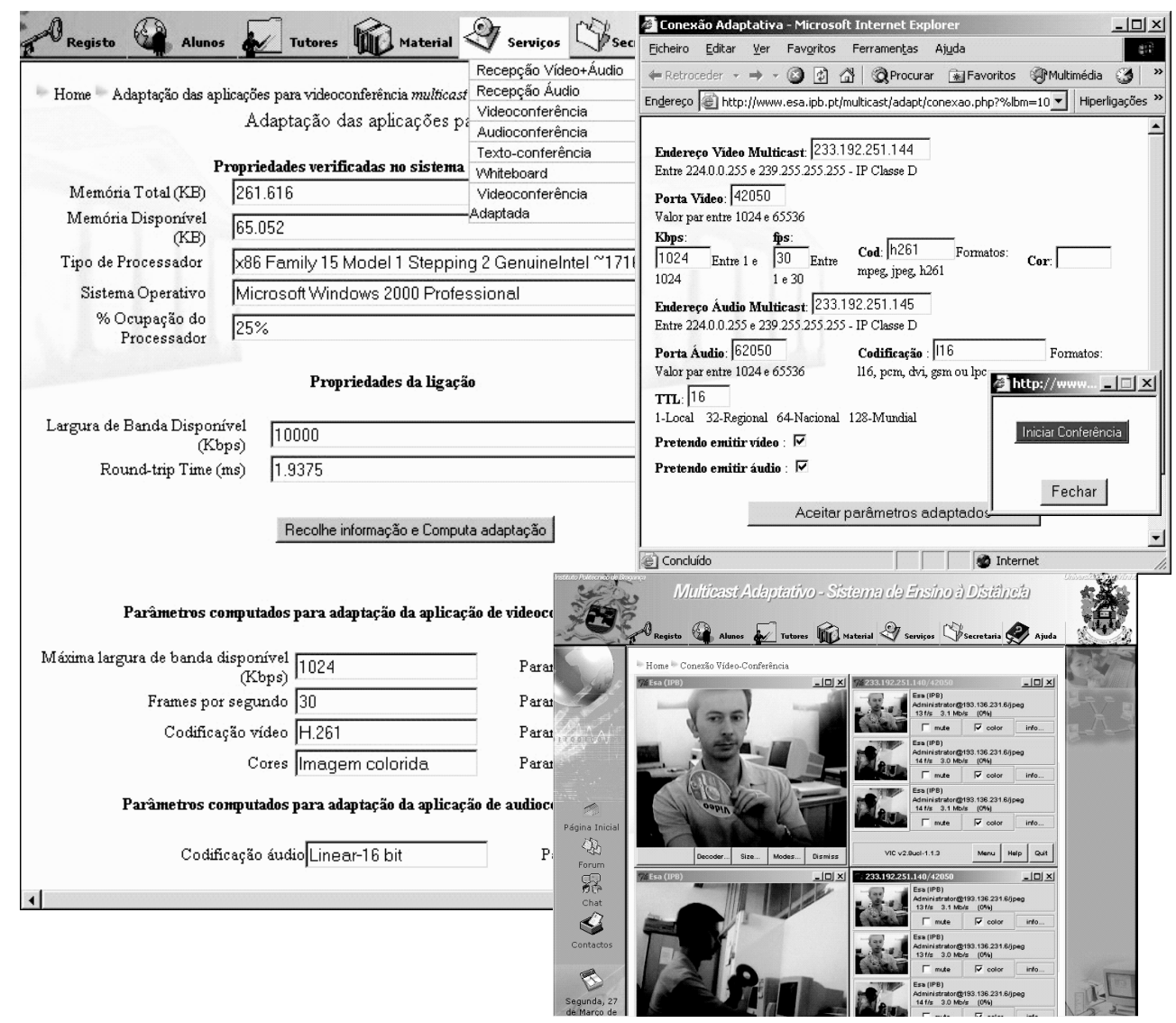

ing to low loss ratios and high reliability. H.263 is especially appropriate for low bandwidth environments.

\section{E-LEARNING SERVICES AND FEATURES}

The developed distance e-learning system presents numerous features providing distinct service levels, such as:

1. Virtual academy, Web-based with refined usability, integrating authentication and services for the e-learning community.
2. Registration, authentication and maintenance of educational agents.

3. Multicast sessions maintenance and scheduling.

4. Access to asynchronous material such as video on demand, slide presentations and other multimedia resources.

5. Interactive multimedia multicast conferences with QoS adaptation.

6. Other multicast tools for shared workspace.

7. Discussion spaces such as forum and multicast chat room. 
This information system incorporates online databases structuring courses, students, tutors and sessions' data. These resources were developed using MySQL/PHP. A Web site congregating all developed application component prototypes is available at www.esa. ipb.pt/multicast.

Certain processes for assessing hardware performance require user's explicit authorization, allowing extended security privileges to applets in order to perform system's inspection and collecting substantial data used by subsequent applets of the control path. The security certificates used in this work are not provided by official entities, but generated by applet compilation tools for testing.

Although the adaptation process is totally transparent, effectively, the users may edit QoS parameters suggested by the system. If editing occurs, correctness and validation are assured by embedded Javascript code for parsing purposes.

All MMC applications need to be previously installed and accessible through the command line interface, configuring the PATH environment variable properly. If we want to transmit audio or video the required equipment must also be ready. Gathering these basic requirements it is possible to participate in e-learning sessions, having adapted QoS in a transparent way, with great usability. Figure 3 integrates a system's screenshots, illustrating step by step, when a QoS adapted video conference is selected from the "Services" menu.

\section{PERFORMANCE STUDY}

Applications that use voice, video streams or multimedia must be carefully managed within an IP network to preserve their operational integrity. Beyond routing improvements, QoS in a multimedia conference needs primarily to deal with several sources with different characteristics, shifting large amount of traffic competing for network capacity. MMC applications may easily absorb all network resources and the subjective quality sensed by users, would remain poor if the available resources are used indiscriminately. As mentioned ear- lier, the adaptation purpose, with e-learning in mind, was to integrate MMC applications with QoS conscience, preserving resources in order to maintain conference quality and improve scalability.

In order to test the framework, different scenarios were simulated and the corresponding resources' consumption verified considering the QoS limitations associated with each QoS mode defined in Table 2. For video conferencing, regarding e-learning purposes, it is widely accepted that reference values correspond to "Maximum quality, few action scene." Bandwidth consumption in vic default mode is 128 $\mathrm{kbps}$. When adaptation is requested, the different adaptation modes use the values charted in Figure 4. For instance, the best quality mode consumes around $400 \mathrm{kbps}$, allowing better image and motion.

Different equipment was also tested in order to validate the rank of the defined adaptation modes. We observed that modern high performance equipment tends to be neutral; in this case adaptation will be influenced overall by network conditions, but with mobile computation in mind, PDAs and cellular phones, CPU performance should not be relegated.

The experimental results were obtained varying the number of new multicast members transmitting voice and video, considering that all the multicast group members are multimedia receivers and transmitters capable using Any Source Multicast (ASM) technology via vic and rat applications. Due to the limited number of multicast monitoring tools publicly available, we use embedded applications resource meters and the Multicast Monitor (www. multicastmonitor.com) to collect and handle the resulting data.

The e-learning system, more concretely the tested prototype showed good performance indicators that validate the architecture model proposed. Because video traffic is quantitatively more representative of resource consumption, it was analyzed preferentially. Figures 4 and 5 exhibit the levels of resource consumption for each QoS mode considered. Here, the overhead introduced by middleware to prepare applica- 
Figure 4. BW needs for each QoS mode

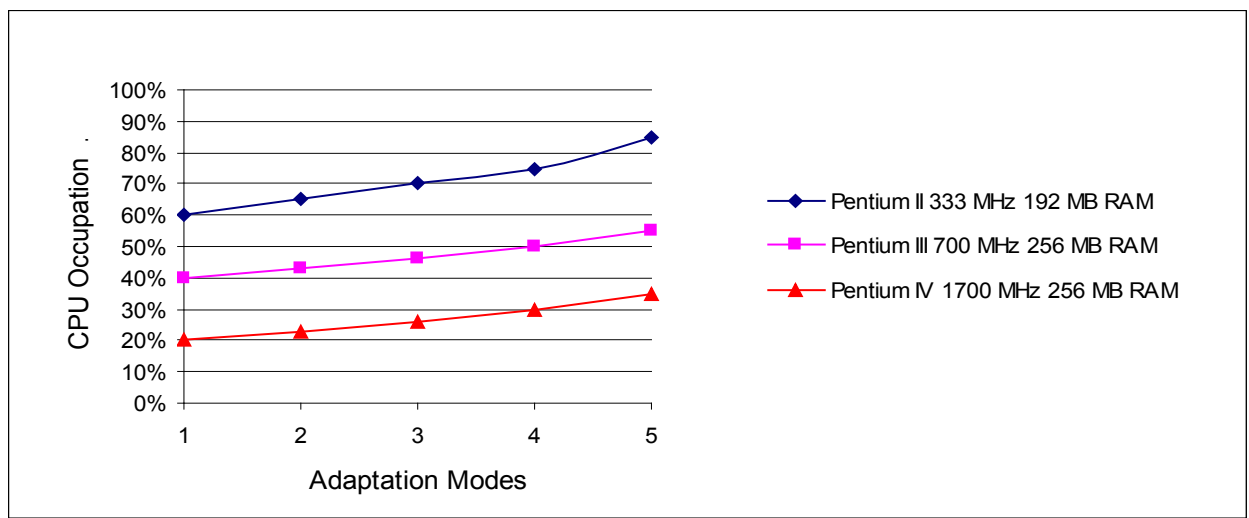

Figure 5. CPU needs for each QoS mode

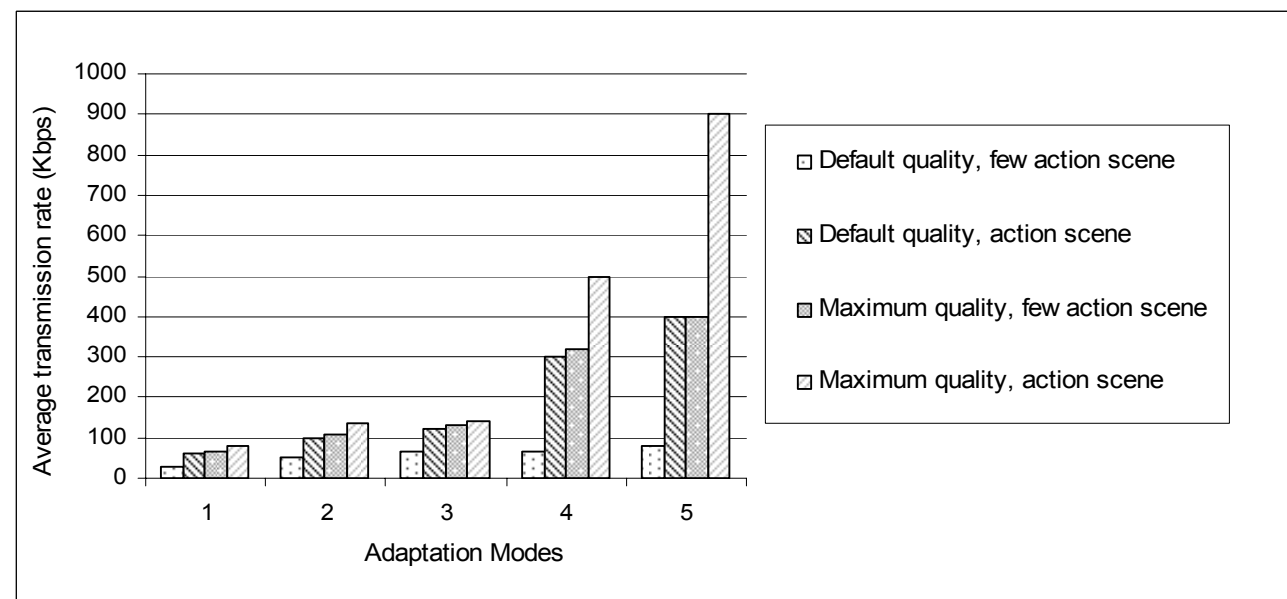

tions is marginal as it occurs before transmission time.

QoS adaptation, when treated systematically in tolerant real-time applications, denotes advantages in group scalability and QoS sustainability in heterogeneous and unpredictable environments such as the Internet and Mbone. Figures 6, 7 and 8 illustrate a comparison between two simulated sessions, the first without QoS adaptation and the second including adaptation managed by the developed middleware layer. The results show that scalability increased, but equally important is the fact that applications may benefit from resource availability that does not occur when using the default applications configuration. When the available resources decrease, the system allocates them to critical parameters. For instance, while the frame rate should not be below $10 \mathrm{fps}$, the image quality may be poor or monochromatic if the contents are correctly perceived.

Limiting bandwidth to applications, not only with explicit parameterization but also choosing the right encoding format for e-learn- 
Figure 6. Linear bandwidth distribution using applications' defaults, no adaptation is used.

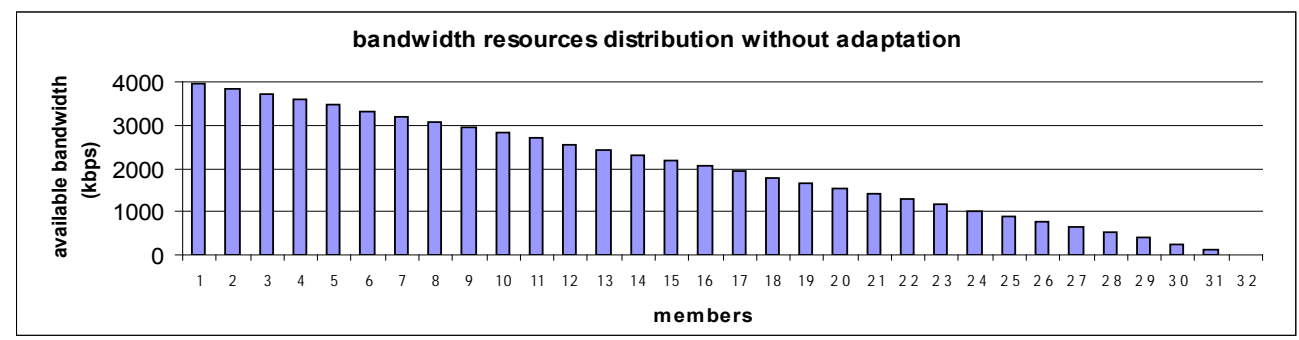

Figure 7. Increasing the number of active group members using adaptation to distribute network resources.

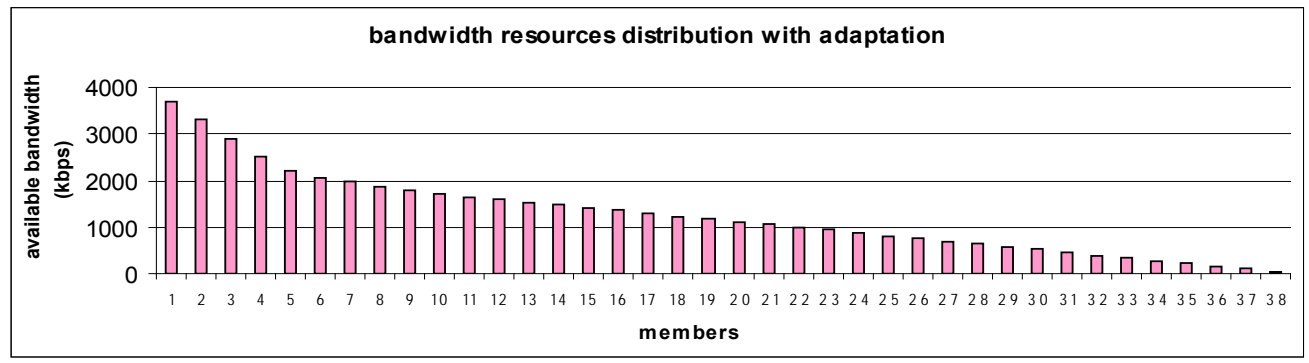

Figure 8. QoS mode adopted by the system facing the available resource conditions

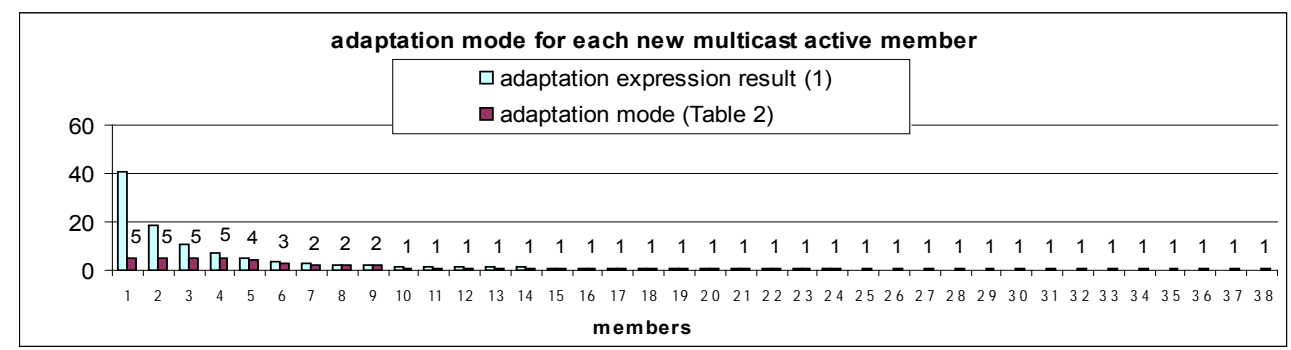

ing sessions, allows efficient resource utilization and proactive usability, avoiding network overload and congestion. If the network load remains high, it is easier to recover if adaptation is used.
The experience with Mbone showed that e-learning groups tend to be small, usually less than 20 members. Effectively, e-learning communities, as in traditional training methods, need a tutor, who is mainly an educational agent 
and not necessarily a learning technologist. Indeed, questions related with communication technologies will not constitute pedagogical limitation if intelligent QoS management autonomy is provided natively or by middleware to applications.

E-learning conferencing specificity requires appropriate video encoding formats able to achieve low loss ratio and fast recovery from congestion. We compared H.261 and Motion JPEG (ITU-JPEG, 1992) performance in experimental sessions, using a modest PC (PIII $0.7 \mathrm{GHz}-256 \mathrm{MB}$ RAM). The results were penalizing for

MJPEG, where loss was about $30 \%$, in opposition to $1 \%$ for H.261.

\section{CONCLUSION AND DISCUSSION}

The goal of this article was, in one topic, to foster "ecological" practices in the Internet when using MMC's applications in e-learning services. The proposed system integrates public domain multicast applications for synchronous media communication, being supervised by a middleware based QoS management framework, intending to preserve the QoS of critical parameters for e-learning session's specificity.

As main contributions, this work:

1. Provides an integrated e-learning environment based on interactive multimedia services with proactive QoS;

2. Improves the usability of MMC applications; and

3. Allows the development of end-to-end QoS-aware multimedia conferences, coordinating resources from network, end-system processing equipment and applications.

Middleware adaptation is a solution that suits the present state of Internet and the requirements of new multimedia distributed applications. We use a middleware layer to manage QoS adaptation in interactive audio and video applications coordinating resource demand, monitoring and adjudication. Substantive results were obtained in group scalability, QoS sustainability and proactive resource utilization.

Comprising multiple sources (even unauthorized ones), ASM involves high complexity and may compromise the success of e-learning conferences. Future work includes the use of Source-Specific Multicast (SSM) (Holbrook \& Cain, 2004) in order to overcome this limitation. The development of new multilayer video encoding formats could also increase the flexibility when using QoS adaptation. When cumulative layers are transmitted avoiding redundancy, using different SSM groups or channels, adaptation can be performed in a transparent way in order to achieve efficient resources utilization (Johanson \& Lie, 2002; Liu, Li \& Zhang, 2004).

\section{ENDNOTE}

' RTT and/or $\mathbf{P}$ values will be, if necessary, assigned to 1 to avoid division by zero. To prevent incongruence, the maximum bandwidth allowed cannot exceed the detected value (B), otherwise the computed mode will suffer cyclic decrements while the excess remains and $\mathbf{M}>1$.

\section{REFERENCES}

Allison, C., Ruddle, A., McKechan, D., \& Michaelson, R. (2001). The architecture of a frameworkfor building distributed learning environments in advanced learning technologies. WI: IEEE Press.

Bolot, J., Crépin, H., \& Garcia, A. (1995). Analysis of audio packet loss in the Internet. France: INRIA.

Bouras, C., \& Gkamas, A. (2003). Multimedia transmission with adaptive QoS based on real time protocols. International Journal of Communications Systems.

Deering, S. (1998). Multicast routing in internetworks and extended LANs. In Proceedings of ACM SIGCOMM (pp 55-64).

Deusdado, S. (2002). Integração Adaptativa de Aplicações Multicast para Conferência Multimédia. Unpublished master thesis, Universidade do Minho. 
Eklund, J., Kay, M., \& Lynch, H. (2003). Elearning: Emerging issues and key trends. Australian Flexible Learning Framework, Australian National Training Authority.

Ferguson, P., \& Huston, G. (1998). Delivering QoS on the Internet and in corporate networks. John Wiley \& Sons.

Hardman, V., Kirstein, P., Sasse, A., Handley, M., \& Watson, A. (1995). RAT, Robust Audio Tool. Retrieved July 27, 2006, from http://www-mice.cs.ucl.ac.uk/multimedia/software/rat/

Holbrook, H., \& Cain, B. (2004). Sourcespecific multicast for IP. Unpublished manuscript.

ITU-JPEG. (1992). JPEG Standard, Information Technology- Digital Compression and Coding of Continuous-Tone Still Images- Requirements and Guidelines, Recommendation T.81, ITU.

ITU-T H.261.(1993). ITU-T Recommendation H.261, Video CODEC For Audiovisual Services At $p \times 64$ kbits.

ITU-T H.263. (1998). ITU-T Recommendation H.263, Video Coding For Low Bitrate Communication.

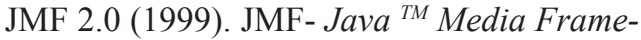
work API Guide, Sun Microsystems, JMF 2.0 FCS.

Johanson, M., \& Lie, A. (2002). Layered encoding and transmission of video in heterogeneous environments. ACM.

Kosiur, D. (1998). IP multicasting: The complete guide to interactive corporate networks. John Wiley \& Sons.

Layaida, O., \& Hagimonte, D. (2002). Dynamic adaptation in distributed multimedia applications. INRIA.

Li, B., Xu, D., Naharstedt, K., \& Liu, J. (1998). End-to-end QoS support for adaptive applications over the Internet. Unpublu- ished manuscript, University of Illinois at Urbana-Champaign.

Liu, J., Li, B., \& Zhang, Y. (2004). An end-toend adaptation protocol for layered video multicast using optimal rate allocation. IEEE Transactions On Multimedia, $6(1)$.

McCanne, S., \& Jacobson, V. (1995). vic: A flexible framework for packet video. In Proceedings of the ACM Multimedia Conference. Retrieved July 27, 2006, from http://www-mice.cs.ucl.ac.uk/multimedia/software/vic/

Miras, D. (2002). A survey of network QoS needs of advanced Internet applications. Unpublished manuscript, University College London.

Mohsin, M., Wong, W., \& Bhutt, Y.(2001). Support for real-time traffic in the Internet, and QoSissues. Unpublished manuscript, University of Texas at Dallas.

Steeples, C., \& Jones, C. (2002) Networked learning: Perspectives and issues. London, UK: Springer.

Thaler, D., \& Handley, M. (2000). On the aggregatability of multicast forwarding state. IEEE INFOCOM.

Tusch, R., Böszörményi, L., Goldschmidt, B., Hellwagner, H., \& Schojer, P. (2004). Offensive and defensive adaptation in distributed multimedia systems. ComSIS, 1(1).

Vandalore, B., Feng, W., Jain, R., \& Fahmy, S. (2001). A survey of application layer techniques for adaptive streaming of multimedia. Real Time Imaging, 7(3), 221-235.

Wu, D., Hou, Y., Zhu, W., Zhang, Y., \& Peha, J. (2001). Streaming video over the Internet: Approaches and directions. IEEE Transactions on Circuits System Video Technology,11(3) 282-300. 
Sérgio Deusdado received his MSc degree in computer science from the University of Minho, Portugal in 2002. Since 2004, he has been a PhD student in the Department of Informatics, at the University of Minho, Portugal. He is currently lecturer in computer science at the Polytechnic Institute of Bragança in Portugal. His research interests include internetworking, groupware, e-learning, information theory and bioinformatics.

Paulo Carvalho was graduated in 1991 and received his PhD degree in computer science from the University of Kent at Canterbury, Canterbury, United Kingdom, in 1997. He is currently assistant professor of computer communications, Department of Informatics, at the University of Minho, Portugal. His main research interests include broadband technologies, multiservice networks and protocols, traffic characterization and modelling and mobile networks. 